\title{
Local computational methods to improve the interpretability and analysis of cryo-EM maps
}

Satinder Kaur ${ }^{1}$, Josue Gomez-Blanco ${ }^{2}$, Ahmad A. Z. Khalifa ${ }^{1}$, Swathi Adinarayanan ${ }^{1}$, Ruben SanchezGarcia $^{3}$, Daniel Wrapp ${ }^{4}$, Jason McLellan ${ }^{3}$, Khanh Huy Bui ${ }^{1}$ and Javier Vargas ${ }^{5}$

${ }^{1}$ Department of Anatomy and Cell Biology, McGill University 3640 Rue University, Montréal, QC, Canada, United States, ${ }^{2}$ Universidad Complutense de Madrid, United States, ${ }^{3}$ United States, ${ }^{4}$ Department of Molecular Biosciences, The University of Texas at Austin, Austin, TX, USA, Hanover, New Hampshire, United States, ${ }^{5}$ Departmento de Óptica, Universidad Complutense de Madrid, Madrid, Spain, United States

High-resolution cryo-electron microscopy (cryo-EM) maps help to visualize and analyze biological macromolecules in their native state as well as to understand their biological functions ${ }^{1}$. Density contrast is as important as a resolution to interpret these maps. Contrast lost at high-resolution in cryo-EM maps results from a resolution-dependent amplitude-falloff, typically called as B-factor. B-factor sharpening approaches ${ }^{2-4}$ allow regaining the lost contrast in the maps. Additionally, effective B-factors help to recognize important map issues such as loss of resolution by macromolecular flexibility, molecular drifting due to charging effects, or possible errors in the reconstruction workflow ${ }^{4-6}$. However, the application of a uniform B-factor for map sharpening to reconstructions showing heterogenous B-factor distributions can lead to 'over sharpening'. In this case, cryo-EM maps are usually distorted and show broken destinies and noise boosting, which may affect the final $a b$ initio modeling. Another important local parameter that was not available yet for cryo-EM map analysis is the map local occupancy, which estimates the presence of an atom at its mean position.

Here, we introduce semi-automated procedures to i) prevent the map distortions while improving the cryo-EM map interpretability at high resolution; ii) attain local B-factors and electron density occupancy maps. The desired outcome of all procedures is to improve the map contrast and propose new local metrics (local B-factor and occupancy map) for improving the interpretation and visibility of cryo-EM reconstruction. The only input requirements are the binary solvent mask, a resolution range, and the map to enhance or analyze.

The proposed approaches are called LocSpiral, LocBSharpen, LocBFactor and LocOccupancy ${ }^{7}$ and have in common the usage of the spiral phase transformation, which can determine the modulation or amplitude map at a different resolution for cryo-EM reconstruction. Specifically, our local map enhancement approach, called LocSpiral, can strongly improve the interpretation and visibility for maps affected by the heterogeneous distribution of local resolutions/SNRs, as shown in several publications[1-4]. During our studies of type IV pili, we discovered nanotube like structures correlated with heterologous expression of the Pseudomonas aeruginosa major type IV pilin, PilA, in some Escherichia coli strains. We have coined these membranous tubular structures P-pods for pilininduced pods based partly on their appearance in negative stain transmission electron microscopy images. Biochemical analysis showed that the major protein inside of P-pods is the inner membrane protein PilA. Because some membranous appendages serve as transport tunnels, we tested horizontal gene transfer in the P-pods; these studies revealed that P-pods are unable to transfer plasmid DNA.

To further characterize the structure of P-pods we carried out both negative stain TEM imaging and cryo-electron tomography. The cells were incubated on LB agar with inducers for 16 hours. Cells were resuspended in LB liquid medium to an $\mathrm{OD}_{600}=0.01$ and spotted on to glow-discharged, Quantifoil R2/1 mesh, gold grids (Quantifoil, Germany) with $5 \mathrm{~nm}$ carbon coating, in a humified chamber for 3.5 hours. Three $\mathrm{mL}$ of BSA-treated $10 \mathrm{~nm}$ colloidal gold (Electron Microscopy Sciences, USA) was applied onto the grids and then plunge-frozen in liquid ethane using a Leica EM GP (Leica Microsystems, Germany). Data collection was performed on a Titan Krios (Thermo Scientific, USA) FEG TEM operated at $300 \mathrm{kV}$ and equipped with a Gatan K3 direct electron detector and a Gatan 
bioquantum energy filter, at the University of Wisconsin-Madison Cryo-EM Research Center. Images were acquired with a pixel size of $0.4603 \mathrm{~nm}$ on the specimen and a total electron does between 120 to $130 \mathrm{e}^{-} / \AA^{2}$. Data were collected at $2^{\circ}$ increments from $-60^{\circ}$ to $+60^{\circ}$ (61 images) with a nominal defocus range of -4.0 to $-6.0 \mu \mathrm{m}$ to enhance contrast of various cell components. Tilt series images were collected using SerialEM [5]. Tomographic reconstructions were generated using IMOD [6] following tilt-series image motion correction by motioncor2 [7] and data were binned two-fold during this process. Neural network based tomogram segmentation models of Ppods were produced with EMAN2 [8].

The resulting 3D reconstructions revealed that $\mathrm{P}$-pods originate at the inner membrane and $\mathrm{P}$-pod extrusion may occur in multiple ways (Figure 1). P-pods may exit the cells through breaks in the outer membrane or by blebbing of the outer membrane which eventually ruptures and releases P-pods (Figure 1). Also, our data indicates that Ppods are comprised of a single lipid-bilayer, do not transport other vesicles, and are typically observed as a string of segmented vesicles once outside of the cell (Figure 2). Correlative light and electron microscopy (CLEM) could provide additional evidence for the localization of PilA within the cell and P-pods and may be the focus of follow up experiments. The biochemical and structural studies presented here reveal that, though similar to nanotubes, Ppods are novel membranous extracellular appendages that spontaneously assemble as the result of PilA overexpression in E. coli and may be a result of protein crowding that leads to positive membrane curvature of the inner membrane.

A

\section{LocSpiral}

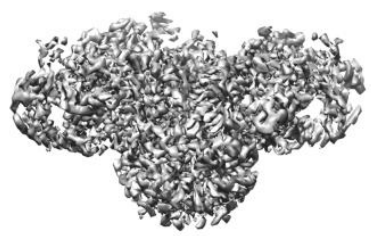

C

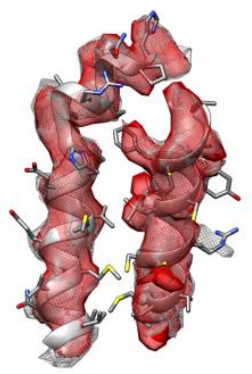

Relion
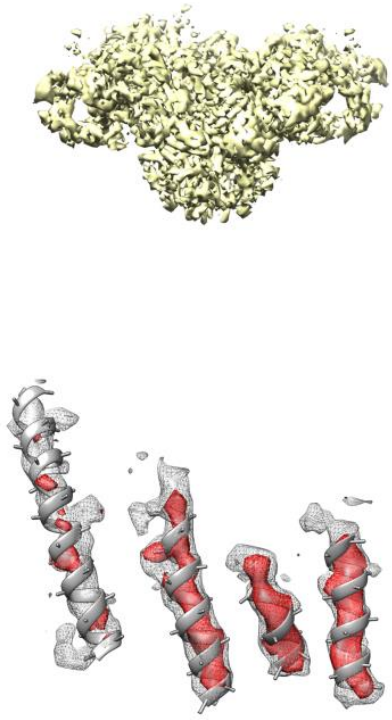

B

LocBFactor
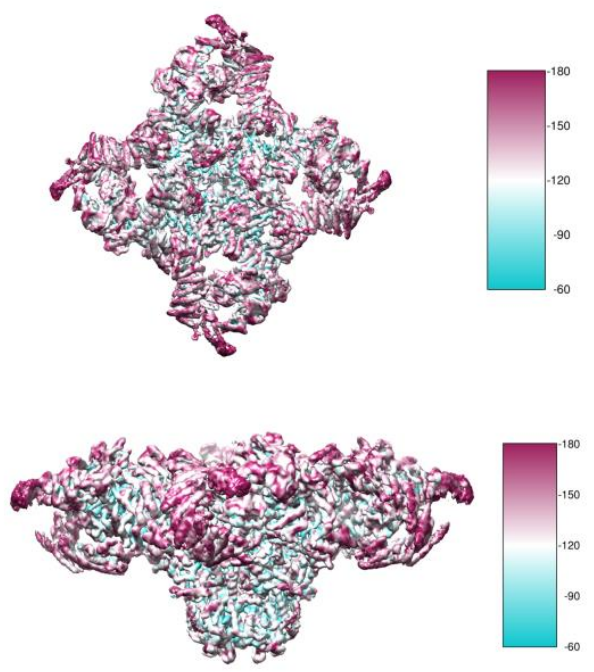

Figure 1. Characterization of P-pod extrusion via blebbing or membrane breach. A) Tomogram reconstruction of an E. coli cell expressing P. aeruginosa PilA protein resulting in blebbing of the outer membrane and localization of p-pods to the bleb. B) Tomogram reconstruction of the PilA expression system resulting in breaks along the outer membrane which allow for extrusion of p-pods. Figures represent a central slice with a thickness of $4.6 \mathrm{~nm}$. 


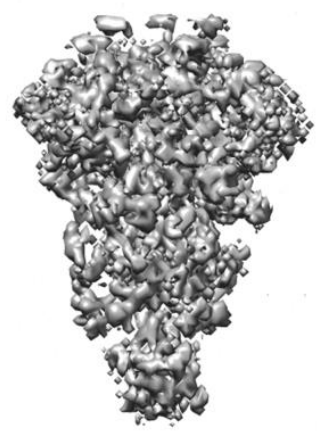

Figure 2. Extracellular P-pod structures. A) Tomogram reconstruction of extracellular p-pods as a string of segmented vesicles and B) a magnified view of a p-pod segment. Figures represent a central slice with a thickness of $4.6 \mathrm{~nm}$.

\section{References}

1. Pal, R.R., et al., Pathogenic E. coli Extracts Nutrients from Infected Host Cells

Utilizing Injectisome Components. Cell, 2019. 177(3): p. 683-696.e18.

2. Weiner, J.H., et al., Overproduction of fumarate reductase in Escherichia coli induces a novel intracellular lipid-protein organelle. J Bacteriol, 1984. 158(2): p. 590-6.

3. Shetty, A., et al., Nanopods: a new bacterial structure and mechanism for deployment of outer membrane vesicles. PloS one, 2011. 6(6): p. e20725-e20725.

4. Wang, F., et al., Structure of Microbial Nanowires Reveals Stacked Hemes that Transport Electrons over Micrometers. Cell, 2019. 177(2): p. 361-369.e10.

5. Mastronarde, D.N., Automated electron microscope tomography using robust prediction of specimen movements. Journal of Structural Biology, 2005. 152(1): p. 36-51.

6. $\quad$ Kremer, J.R., D.N. Mastronarde, and J.R. McIntosh, Computer Visualization of Three-Dimensional Image Data Using IMOD. Journal of Structural Biology, 1996. 116(1): p. 71-76.

7. Zheng, S.Q., et al., MotionCor2: anisotropic correction of beam-induced motion for improved cryo-electron microscopy. Nature methods, 2017. 14(4): p. 331-332.

8. Chen, M., et al., Convolutional neural networks for automated annotation of cellular cryo-electron tomograms. Nature methods, 2017. 14(10): p. 983-985.

9. This research was supported by funds from the University of Wisconsin-Madison, National Institutes of Health (R01GM104540 and R01GM104540-03S1) to E.R.W. J.C.S. was supported in part by the Biotechnology Training Program, T32GM135066. All EM data was collected at the University of Wisconsin-Madison, Department of Biochemistry Cryo-EM Research Center. The authors gratefully acknowledge use of facilities and instrumentation at the UW-Madison Wisconsin Centers for Nanoscale Technology (wcnt.wisc.edu) partially supported by the NSF through the University of Wisconsin Materials Research Science and Engineering Center (DMR-1720415). 